\title{
Drum zahle, wer sich ewig bindet: Eine Analyse der Gebührenhöhe für eingetragene Lebenspartnerschaften in Baden-Württemberg
}

\author{
Marc Debus / Christoph Knill / Jale Tosun
}

Why Gay Couples Pay More: Determinants of the Level of Fees for Same-Sex Unions in Baden-Württemberg

Abstract: Since 2001 gay couples in Germany are given the possibility to legalize their relationship through the registration as a same-sex union. While 15 German states adopted uniform fees for the legal registration of same-sex couples, the state government of BadenWuerttemberg opted for an individual approach: Each county decides about the fee level that a gay couple has to pay when registering at the district office. As a consequence, there is a remarkable variation in the levels of registration fees across the individual regional units of the state. In this study, we address this variation in registration fees and develop an explanatory model that builds on central concepts of public policy analysis and political economy. Our analysis reveals that the varying levels of registration fees for same-sex unions are determined by the regional political culture, economic problem pressure and the partisan composition of the regional parliaments. In this sense, an increasing representational strength of socially progressive political parties entails lower fee levels.

Key words: Same-Sex Unions, State of Baden-Wuerttemberg, Registration Fees, Partisan Politics, Political Culture, Economic Problem Pressure

Schlagwörter: Eingetragene Lebenspartnerschaft, Baden-Württemberg, Gebührenhöhe, Parteipolitik, Politische Kultur, ökonomischer Problemdruck

\section{Einleitung}

Im Sommer 2010 versuchten Interessengruppen, die für die Rechte homosexueller Menschen eintreten, auf eine offensichtliche Diskriminierung gleichgeschlechtlicher Paare in Baden-Württemberg hinzuweisen. Im Gegensatz zu allen anderen 15 Bundesländern werden im Südwesten der Republik eingetragene Lebenspartnerschaften nicht vor den Standesämtern geschlossen. Vielmehr wird den Landkreisen und kreisfreien Städten durch ein Landesgesetz vorgeschrieben, die Kreisoder Stadtverwaltung mit dieser Aufgabe zu betrauen. Letzteres führt dazu, dass die Verpartnerung oftmals nicht in angemessenen Räumlichkeiten wie denen des Standesamts, sondern improvisiert in Bereichen der Kreisverwaltung - in manchen Fällen etwa der Kfz-Zulassungsstellen - geschlossen werden müssen (vgl. Die Tageszeitung vom 29.07.2010; Süddeutsche Zeitung vom 10.08.2010). 
$\mathrm{Zu}$ dieser symbolischen Ungleichbehandlung kommt ein weiterer, materieller Faktor hinzu, der den Unterschied zwischen Ehe und Lebenspartnerschaft klar manifestiert: Die von 1996 bis 2011 aus CDU und Freien Demokraten bestehende Landesregierung Baden-Württembergs hat den Beschluss gefasst, den Kreisen und kreisfreien Städten die Entscheidung über die Höhe des Gebührensatzes zu überlassen. Dies hat zur Folge, dass die Gebühr, die anfällt, wenn man eine eingetragene Lebenspartnerschaft in Baden-Württemberg schließen will, zwischen Kreisen und kreisfreien Städten mitunter deutlich variiert. So betragen die Anmeldekosten in der Stadt Heidelberg $40 €$ und sind damit gleich hoch wie die Grundgebühren, die bei der Schließung einer Ehe anfallen. Der Zollernalbkreis hingegen verlangt mit Stand von Oktober 2010 für die Schließung einer gleichgeschlechtlichen Partnerschaft $238 €$, das heißt einen Betrag von $198 €$ mehr an Gebühren als für die Anmeldung im Rahmen einer Eheschließung.

Womit lassen sich diese zwischen den Landkreisen und kreisfreien Städten Baden-Württembergs bestehenden Unterschiede in der Gebührenhöhe für eingetragene Lebenspartnerschaften erklären? Spielen ideologisch-programmatische Faktoren eine Rolle? Dies legt zumindest der Regierungswechsel in Baden-Württemberg in Folge der Landtagswahlen vom März 2011 nahe. So ist laut Koalitionsabkommen von Bündnis 90/Die Grünen und SPD vorgesehen, die Zuständigkeit für die Schließung von Lebenspartnerschaften auf die Standesämter zu übertragen und einheitliche, mit der Eheschließung übereinstimmende Gebührensätze einzuführen, was eine grundlegende Reform der noch bestehenden Regelungen impliziert (vgl. Bündnis 90/Die Grünen und SPD Baden-Württemberg 2011, S. 74).

Dieser Beitrag geht unter Zuhilfenahme policy-analytischer und politökonomischer Theorien der Varianz in den Gebührenhöhen nach und erörtert zudem die potenzielle politische Steuerungswirkung von Verwaltungsgebühren. Hierbei wird wie folgt vorgegangen: Zunächst diskutieren wir die Regelung der eingetragenen Lebenspartnerschaft vor dem Hintergrund der Moralpolitik als einem Politikfeld, das parteipolitische Akteure vielfach zur inhaltlichen Profilierung und damit zur Mobilisierung verschiedener Wählersegmente nutzen (vgl. etwa Haider-Markel 1999; Mooney u. Lee 2000; Mooney 2001; Ainsworth u. Hall 2010). Dabei gehen wir sowohl auf die Entwicklung der Regulierung gleichgeschlechtlicher Lebenspartnerschaften bzw. der Homosexualität im internationalen Vergleich und in Deutschland insbesondere ein als auch auf Ergebnisse, die sich aus der Implementationsforschung und der Literatur zur politischen Steuerung ergeben. Im darauffolgenden, dritten Abschnitt werden auf der Grundlage von Theorien zum Problemdruck, der regionalen politischen Kultur und zum Handeln von parteipolitischen Akteuren in modernen Demokratien Erwartungen hinsichtlich der Höhe der Gebühren für eingetragene Lebenspartnerschaften entwickelt, die im fünften Abschnitt empirisch getestet werden. Zuvor muss jedoch auf die Operationalisierung der abhängigen und erklärenden Variablen sowie auf die Quellen der hier verwendeten Daten eingegangen werden. Die Schlussbetrachtung fasst die gewonnen Erkenntnisse zusammen und verweist darauf, dass nicht zuletzt mit Blick auf die politische Steuerungswirkung von Verwaltungsgebühren ein erheblicher Forschungsbedarf besteht, der weit über den hier betrachteten Fall hinausgeht. 


\section{Forschungsstand}

\subsection{Eingetragene Lebenspartnerschaften als Bereich der Moralpolitik}

Das Gesetz über die eingetragene Lebenspartnerschaft (kurz: Lebenspartnerschaftsgesetz; LPartG) ermöglicht zwei Menschen gleichen Geschlechts die Begründung einer Verbindung mit gesetzlich geregelten Rechtsfolgen, die von einer Behörde beurkundet wird. Die Lebenspartnerschaft ist in Deutschland die einzige Möglichkeit, einer gleichgeschlechtlichen Beziehung einen rechtlichen Rahmen zu geben, der - abgesehen vom verfassungsrechtlichen Status sowie steuerlichen Regelungen - dem Institut der Ehe weitgehend gleichgestellt ist. Die Besonderheit, welche die Regulierung der „Homo-Ehe“, wie die Lebenspartnerschaft umgangssprachlich genannt wird, mit anderen, auf den ersten Blick so unterschiedlichen Themen wie Abtreibung, Sterbehilfe oder Glücksspiel teilt, liegt darin, dass diese Policies typischerweise mit Kontroversen über moralische Grundprinzipien einer Gesellschaft einhergehen können.

Die Regulierung gleichgeschlechtlicher Beziehungen wird daher in der policyanalytischen Diskussion einem spezifischen Politiktypus zugeordnet, der unter dem Stichwort Moralpolitik firmiert (Mooney u. Lee 2000; Mooney 2001). Kennzeichnend für diesen Politiktypus ist der Umstand, dass politische Entscheidungsprozesse in starkem Maße durch gesellschaftliche Wertkonflikte charakterisiert sind und nicht, wie etwa im Falle (re-)distributiver oder regulativer Politiken, durch Konflikte über materielle Ressourcen. Aufgrund der dominanten Rolle von Wertkonflikten wird darauf verwiesen, dass Moralpolitiken spezifische Charakteristika aufweisen. Hierzu zählen insbesondere ein vergleichsweise hoher Grad gesellschaftlicher Mobilisierung, eine hohe Polarisierung zwischen den Teilnehmern am politischen Prozess, eine hohe Responsivität parteipolitischer Akteure gegenüber Veränderungen der öffentlichen Meinung sowie vielfach eingeschränkte Möglichkeiten der politischen Kompromissfindung im Wege von Verhandlungslösungen und Tauschprozessen (vgl. Meier 1994; Haider-Markel 1999; Tatalovich u. Smith 2001; Patton 2007; Mooney u. Schuldt 2008; Ainsworth u. Hall 2010).

Ländervergleichende Studien zu Ursachen moralpolitischen Wandels fehlen weitgehend (eine Ausnahme bildet Fink 2007, 2008). Vielmehr erfolgt bislang eine starke Konzentration auf den angloamerikanischen Kontext (vgl. etwa Tatalovich u. Smith 2001; Ainsworth u. Hall 2010). Hinzu kommt eine Konzentration auf einzelne Felder, insbesondere Abtreibung und Sterbehilfe, während zum hier interessierenden Thema Homosexualität und speziell gleichgeschlechtliche Partnerschaften nur wenige Untersuchungen vorliegen. Zu erwähnen sind hier die Studien von Wald et al. (1996) sowie Haider-Markel und Meier (1996), welche die Politiken zur Nichtdiskriminierungspolitik Homosexueller im US-amerikanischen Kontext vergleichend analysieren. Einen Überblick über die Homosexuellen-Bewegung in der Bundesrepublik gibt die Abhandlung von Haunss (2004); die Entwicklung der deutschen Nichtdiskriminierungspolitik wird von Gammerl (2010) nachgezeichnet. Insgesamt verweisen die wenigen bislang vorliegenden Studien darauf, dass die Regulierung und Ermöglichung gleichgeschlechtlicher Lebenspartnerschaften international als Element einer zunehmenden rechtlichen 
Gleichstellung homosexueller und heterosexueller Menschen ist. Dieser Trend der Liberalisierung von (Homo-)Sexualität war und ist nicht nur in Deutschland, welches diesbezüglich im internationalen Vergleich eher nachholend reguliert hat, stets durch einen in starkem Maße von konfligierenden gesellschaftlichen Wertvorstellungen geprägten politischen Prozess gekennzeichnet.

Das Lebenspartnerschaftsgesetz wurde am 16. Februar 2001 verabschiedet und trat zum 1. September 2001 in Kraft. Der damit geschaffene rechtliche Rahmen für gleichgeschlechtliche Beziehungen ist in seiner Ausgestaltung dem Rechtsinstitut der Ehe im Hinblick auf bürgerlich-rechtliche Angelegenheiten weitgehend gleichgestellt. Dessen ungeachtet bestehen in verschiedener Hinsicht gravierende Unterschiede zur Ehe. Letztere beziehen sich insbesondere auf die verfassungsrechtliche Schutzgarantie, von der die eingetragene Lebenspartnerschaft im Gegensatz zur Ehe nicht erfasst wird. Überdies verbleiben Diskrepanzen in der einkommenssteuerrechtlichen Behandlung, wo Ehepartnern, nicht jedoch eingetragenen Lebenspartnern besondere Begünstigungen (prominenteste Beispiele sind das Ehegattensplitting und das Steuerklassenwahlrecht) zugestanden werden (vgl. etwa Bruns u. Kemper 2005).

Die deutsche Gesetzeslage liegt damit in etwa auf einer Linie mit Dänemark, Finnland, Frankreich, Großbritannien, Österreich und der Schweiz, die ähnliche Regelungen vorsehen. Eine vollständige Gleichstellung zwischen Ehe und Lebenspartnerschaft besteht dagegen in Belgien, den Niederlanden, Norwegen, Portugal, Schweden und Spanien, während in einigen osteuropäischen Staaten - unter anderem in Polen - sogar ein gesetzliches Verbot solcher Partnerschaften besteht (Waaldijk 2004; Boele-Woelki 2008). Die Verabschiedung des Lebenspartnerschaftsgesetzes ist in Deutschland wie auch vielen anderen Staaten im Zusammenhang mit einer inkrementell verlaufenden Entwicklung der Entkriminalisierung der Homosexualität zu sehen. In der Bundesrepublik kam es erst ab 1969 zu einer zögerlichen Liberalisierung, nachdem nach dem Zweiten Weltkrieg die sehr restriktiven strafrechtlichen Vorschriften aus der Zeit des nationalsozialistischen Regimes unverändert übernommen worden waren und auch die Rechtsprechung des Bundesverfassungsgerichts nahtlos an diese Rechtspositionen angeknüpft hatte (Stümke u. Finkler 1981, S. 140-149; Stümke 1989, S. 132-140; Moeller 1994, S. 405-410; Steinke 2005, S. 61). Erst 1994 kam es im Zuge der Wiedervereinigung und der damit erforderlichen Rechtsanpassung zu einer völligen Entkriminalisierung der Homosexualität (Gammerl 2010, S. 8-9). Gleichzeitig gab es verstärkt Bestrebungen, eine rechtliche Basis für die bis in die 1980er-Jahre als sittenwidrig eingestuften gleichgeschlechtlichen Partnerschaften zu etablieren. Diese Bestrebungen waren nicht nur inspiriert von Entwicklungen in Vorreiterländern wie Dänemark, wo entsprechende Regelungen bereits 1989 verabschiedet worden waren, sondern auch durch verbesserte Strukturen und Ressourcen homosexueller Interessenverbände und verschiedene Gesetzesentwürfe, die seitens der Grünen im Deutschen Bundestag eingebracht worden waren.

Die Chance für die Umsetzung dieser Pläne bot sich dann mit dem Regierungswechsel 1998. Das Lebenspartnerschaftsgesetz wurde im November 2000 vom Bundestag mit den Stimmen der Regierungsparteien gegen die Stimmen von Union und FDP beschlossen. Die beschlossenen Regelungen blieben jedoch hinter 
dem Regierungsentwurf zurück, weil die zustimmungspflichtigen Elemente des Entwurfs im Bundesrat durch die unionsregierten Länder blockiert wurden. In der parlamentarischen Diskussion manifestierte sich ein fundamentaler Konflikt zwischen Regierung und Opposition, der in starkem Maße durch unterschiedliche Wertvorstellungen geprägt war und letztlich für den gesamten Bereich der Homosexualitätspolitik in Deutschland charakteristisch war und auch nach wie vor ist. Insbesondere die unionsregierten Länder sahen in der eingetragenen Lebenspartnerschaft eine Infragestellung der staatlichen Ordnung, in der Ehe und Familie besonderen Schutz genießen. Die zu diesem Zeitpunkt von CDU bzw. CSU allein regierten Länder Bayern, Sachsen und Thüringen klagten nach dem Bundestagsbeschluss vor dem Bundesverfassungsgericht. Sie verwiesen darauf, dass es laut Grundgesetz kein vergleichbares Rechtsinstitut neben der Ehe geben dürfe. Das Bundesverfassungsgericht verwarf diese Argumentation im Jahr 2002. Die Position von CDU und CSU, aber auch von Teilen der SPD war überdies erheblich von dem Standpunkt der Kirchen beeinflusst, welche in der rechtlichen Anerkennung gleichgeschlechtlicher Lebenspartnerschaften eine fundamentale Gefährdung von Ehe und Familie sehen (Stümke 1989, S. 136; Steinke 2005, S. 63).

\subsection{Implementationsforschung}

Wenngleich in der Implementationsforschung vielfach darauf verwiesen wird, dass die effektive formale und praktische Umsetzung eines Gesetzes eher die Ausnahme als die Regel darstellt (Pressman u. Wildavsky 1973; May 2003; Winter 2003), erscheint gerade das Lebenspartnerschaftsgesetz als Maßnahme, welche in dieser Hinsicht keine besonderen Probleme aufwerfen sollte. Dies liegt zum einen daran, dass es sich hier um eine vergleichsweise einfache Regelungsmaterie mit klar definierten Tatbestandsmerkmalen und Rechtsfolgen handelt. Damit hebt sich dieser Fall sehr deutlich von Regulierungsbereichen ab, in denen unbestimmte Rechtsbegriffe, breite Ermessensspielräume sowie hohe technische, wissenschaftliche oder rechtliche Komplexität mit oftmals großen Umsetzungsschwierigkeiten einhergehen. Dies gilt etwa in besonderem Maße für die Umweltpolitik, aber auch für viele Bereiche der wirtschaftlichen Regulierung (Mayntz 1983; Knill 2006; Knill u. Tosun 2010).

Auf der anderen Seite hebt sich der Fall des Lebenspartnerschaftsgesetzes von vielen anderen Bereichen gerade dadurch ab, dass die Adressaten, namentlich homosexuelle Paare, in starkem Maße an einer effektiven Umsetzung des Gesetzes interessiert sind - im Gegensatz etwa zu Industriebetrieben mit Blick auf umweltoder arbeitsschutzrechtliche Vorgaben, die möglicherweise auf eine eher laxe Auslegung oder gar faktische Nichtimplementation drängen. Mit anderen Worten: Von der Interessenkonstellation her sollte das Lebenspartnerschaftsgesetz gewissermaßen ,selbst-implementierend' sein, nicht zuletzt auch deswegen, weil der administrative Umsetzungsaufwand als vergleichsweise gering erachtet werden kann.

Dieser Sichtweise steht jedoch der Umstand entgegen, dass gerade für den Bereich der Moralpolitik systematische Implementationsprobleme vermutet werden können. So wird betont, dass die Präsenz stark ausgeprägter Wertekonflikte mit 
einer geringen Folgebereitschaft derjenigen Akteure einhergehen könnte, welche sich im Prozess der Politikformulierung mit ihrer Position nicht durchsetzen konnten (Mooney 2001). Da - wie oben ausgeführt - solche Probleme im Fall des Lebenspartnerschaftsgesetzes kaum von den Adressaten ausgehen dürften, richtet sich der Blick in diesem Zusammenhang insbesondere auf die für die Umsetzung des Gesetzes zuständigen Behörden und deren mögliche Handlungsspielräume.

Die Umsetzung des Lebenspartnerschaftsgesetzes fällt in die Zuständigkeit der Länder, welche gemäß $\$ 23$ (1) LPartG selbst darüber entscheiden, welche Behörden sie mit der Durchführung betrauen. Diese Regelung setzten die Bundesländer im Rahmen der parlamentarischen Beratungen über das Lebenspartnerschaftsgesetz durch. Die Länder haben diese Aufgabe größtenteils an die Standesämter übertragen. Lediglich in Bayern, Baden-Württemberg und Thüringen bestanden zunächst bzw. bestehen nach wie vor hiervon abweichende Regelungen. In Bayern konnten Lebenspartnerschaften zu Beginn nur vor Notaren geschlossen werden. Dies wurde im Sommer 2009 - maßgeblich aufgrund der geänderten parteipolitischen Zusammensetzung der bayerischen Landesregierung, die seit Herbst 2008 aus CSU und FDP gebildet wird - dahingehend geändert, dass die Schließung einer Lebenspartnerschaft sowohl vor Notaren als auch vor Standesämtern möglich ist (vgl. Christlich-Soziale Union und Freie Demokratische Partei Bayern 2008, S. 62-63). In Baden-Württemberg sind hingegen laut $\$ 1$ des Gesetzes zur Ausführung des Lebenspartnerschaftsgesetzes (LPartAusfG) vom 20. Juni 2002 die Kreisund Stadtverwaltungen und explizit nicht die Standesämter zuständig. In Thüringen galt bis zum 31. Dezember 2010 eine ähnliche Regelung. Die dort amtierende Regierungskoalition aus Christ- und Sozialdemokraten hat dies mit einem Gesetz jedoch abgeändert, sodass seit Januar 2011 eingetragene Lebenspartnerschaften nunmehr auch in Thüringen vor dem Standesamt geschlossen werden müssen (Christlich Demokratische Union Thüringen und Sozialdemokratische Partei Deutschlands, Landesverband Thüringen 2009, S. 48). Der baden-württembergische Fall ist insofern von besonderem analytischem Interesse, als hier - im Gegensatz zu Thüringen - keine landeseinheitliche Umsetzung gegeben ist, sondern lokale Abweichungen derzeit noch möglich sind. Damit bleibt zu klären, wie solche Abweichungen im konkreten Fall aussehen können. Diesem Aspekt wenden wir uns im folgenden Abschnitt zu.

\subsection{Verwaltungsgebühren als Instrument politischer Steuerung?}

Neben der Frage, welche Ämter in den baden-württembergischen Kreisen mit der Durchführung beauftragt sind, ist für unseren Zusammenhang insbesondere von Bedeutung, dass - im Gegensatz zur landesweit einheitlichen Festlegung von Gebühren für standesamtliche Leistungen - die kommunale Ebene bezüglich der Höhe der erhobenen Verwaltungsgebühren über einen breiten Umsetzungsspielraum verfügt.

Gebühren bezeichnen dabei generell Vergütungen, welche von den Zahlungspflichtigen für unmittelbar von ihnen veranlasste öffentliche Leistungen erhoben werden. Nach der Rechtsprechung des Bundesverfassungsgerichts ist eine Gebühr eine öffentlich-rechtliche Geldleistung, die aus Anlass individuell zurechenbarer, 
öffentlicher Leistungen dem Gebührenschuldner einseitig auferlegt wird und dazu bestimmt ist, in Anknüpfung an diese Leistung deren Kosten ganz oder teilweise zu decken (vgl. BVerfGE 50: 217, S. 226). Generell wird dabei eine Unterscheidung zwischen Benutzungsgebühren, welche von der Inanspruchnahme einer Einrichtung abhängen, und - den in unserem Fall vorliegenden - Verwaltungsgebühren, die für eine Amtshandlung erhoben werden, vorgenommen.

Die Gebührenhöhe und mögliche diesbezügliche Spielräume sind in den Verwaltungskostengesetzen der Länder geregelt. Im Falle Baden-Württembergs ist hierfür das Landesgebührengesetz relevant. Generell sollen Gebührensätze zwei Grundsätzen entsprechen: Kostendeckung und Äquivalenz. Nach dem Prinzip der Kostendeckung soll der Gesamtertrag der erhobenen Abgaben die Gesamtkosten des Gemeinwesens für den betroffenen Verwaltungszweig nicht oder höchstens geringfügig übersteigen. $\mathrm{Zu}$ diesem Gesamtaufwand sind auch angemessene Rückstellungen, Abschreibungen und Reserven hinzuzurechnen. Das Prinzip der Äquivalenz besagt, dass die erhobene Abgabe im Einzelfall zum objektiven Wert der Leistung in keinem offensichtlichen Missverhältnis stehen darf und sich in vernünftigen Grenzen bewegen muss. Im Unterschied zum Kostendeckungsprinzip bezieht sich das Äquivalenzprinzip somit immer nur auf das Verhältnis von Abgabe und Leistung. Es ist daher zur Festsetzung von Gebühren zwingend notwendig, den durchschnittlichen Verwaltungsaufwand, der durch eine Amtshandlung entsteht, zu ermitteln und den durchschnittlichen Wert bzw. Nutzen oder die Bedeutung der Amtshandlung für den Leistungsempfänger abzuschätzen.

Da davon ausgegangen werden kann, dass sich die Kostensituation für jeden Land- bzw. Stadtkreis relativ einheitlich darstellt, sollten sich mögliche Gebührenunterschiede allenfalls in der unterschiedlichen Bewertung des jeweiligen Nutzens ergeben. Grundsätzlich können in Fällen, in denen es sich um begünstigende Amtshandlungen handelt (d. h. öffentliche Leistungen, die dem Leistungsempfänger die Wahrnehmung eines rechtlichen, wirtschaftlichen oder sonstigen Vorteils ermöglichen), Gebühren erhoben werden, welche die administrativen Kosten übersteigen ( $\mathbb{S} 7$ (2) Landesgebührengesetz Baden-Württemberg). Vor diesem Hintergrund sind die jeweiligen Behörden daher durchaus in der Lage, innerhalb enger Grenzen rechtlicher Angemessenheit Gebühren zu erheben, welche ihre administrativen Kosten übersteigen. Damit eröffnen sich zweifelsohne Handlungsspielräume, bei deren Ausschöpfung unterschiedliche politische Erwägungen einfließen können.

Dass in Baden-Württemberg die Kreis- und Stadtverwaltungen, die im Regelfall über keine geeigneten Räumlichkeiten verfügen, und nicht wie in allen anderen Bundesländern die Standesämter (und in Bayern zusätzlich die Notare) für das Schließen einer Lebenspartnerschaft verantwortlich sind, kann zudem als Form „symbolischer Politik“ (vgl. Sarcinelli 1989; Edelman 2005; Göhler 2005; Cohen u. Langenhan 2009) gesehen werden, die das Ziel hat, den Unterschied zwischen Ehe und Lebenspartnerschaft zu verdeutlichen. Die in vielen baden-württembergischen Landkreisen im Vergleich zur Eheschließung höheren Gebühren für das Schließen einer Lebenspartnerschaft verstärken den letztgenannten Aspekt noch zusätzlich: Durch höhere Kosten wird nicht nur symbolisiert, dass eine Lebenspartnerschaft etwas anderes darstellt als eine Ehe, sondern es wird seitens des 
Staates durch eine Form „weicher Steuerung“ (Göhler et al. 2010) in manchen Landkreisen auch versucht, die Hürden zum Eingehen einer Lebenspartnerschaft in Form von höheren Gebühren zu erhöhen. ${ }^{1} \mathrm{Da}$ das baden-württembergische Gesetz zur Ausführung des Lebenspartnerschaftsgesetzes nicht nur vorsieht, dass Anträge auf Schließung einer Lebenspartnerschaft bei Landratsämtern und Stadtverwaltungen und nicht bei den Standesämtern abzugeben sind $(\mathbb{S} 1$ (1) LPartAus$\mathrm{fG})$, sondern auch, dass die zuständige Behörde diejenige sein muss, in deren $\mathrm{Zu}$ ständigkeitsbereich die Antragsteller ihren Hauptwohnsitz haben ( $\mathbb{S} 1$ (2) LPartAusfG), besteht für gleichgeschlechtliche Paare ausdrücklich auch nicht die Möglichkeit, in einen anderen Land- oder Stadtkreis mit niedrigeren Gebühren auszuweichen.

Die Frage, ob und inwieweit Verwaltungsbehörden Gebühren veranschlagen, die die eigentlichen Kosten des Verwaltungsvorgangs übersteigen und welche Faktoren hierbei ausschlaggebend sind, ist bislang von der Politikwissenschaft wenig beachtet worden. Daher zielen unsere nachfolgenden theoretischen und empirischen Überlegungen darauf ab, einen ersten Beitrag zum Abbau dieses Forschungsdesiderats zu leisten.

\section{Theoretische Erklärungsansätze und Hypothesen}

Folgt man politisch-soziologischen Studien zum Wahlverhalten und Parteienwettbewerb in der Bundesrepublik, so markieren gesellschaftspolitische Themen und Konflikte zentrale Merkmale der politischen Auseinandersetzung, mit denen Parteien und Kandidaten ihre jeweilige Anhängerschaft mobilisieren können (vgl. Lipset u. Rokkan 1967; Pappi u. Shikano 2002). Dazu zählen etwa Themen der Innen- und Rechtspolitik, wie beispielsweise der von der CDU im hessischen Landtagswahlkampf 2008 thematisierte Umgang mit kriminellen Jugendlichen, die einen Migrationshintergrund haben (vgl. Schmitt-Beck u. Faas 2009). Als ein weiteres Beispiel dient die Unterschriftensammlung der CDU gegen die Einführung der so genannten „doppelten Staatsbürgerschaft" durch die rot-grüne Bundesregierung, die erfolgreich zur Mobilisierung konservativ ausgerichteter Wähler bei der Landtagswahl in Hessen im Februar 1999 beitrug (vgl. etwa Schmitt-Beck 2000). Auch moralisch aufgeladene Themen wie die Regelung von Abtreibung, Sterbehilfe oder die rechtliche Stellung gleichgeschlechtlicher Partnerschaften stellen Möglichkeiten für politische Akteure dar, ihre gesellschaftspolitische Haltung - sei sie progressiv oder konservativ ausgerichtet - für die potenzielle Wählerschaft wie auch für die Parteianhänger zu verdeutlichen. So findet sich in vielen Wahlprogrammen von CDU und CSU auf Bundes-, Europa- und Landesebene ein Bekenntnis zum Schutz des ungeborenen Lebens (vgl. etwa Fink 2008), obwohl dieses Thema entweder nicht auf der tagespolitischen Agenda steht und/oder die

1 In Anbetracht der Gesamtkosten einer Hochzeits- oder Verpartnerungsfeier machen die Gebühren in der Regel nur einen kleinen Anteil aus. Dennoch ist der Effekt eines unterschiedlichen Ortes der Durchführung des Verwaltungsvorgangs und einer höheren Gebühr aus der Perspektive der Personen, die eine Lebenspartnerschaft eingehen wollen, eindeutig: Eine Partnerschaft wird in symbolischer Form gegenüber der Ehe abgewertet. 
entsprechende politische Ebene, für die das programmatische Dokument verfasst wurde, gar keine Kompetenzen besitzt, gegebenenfalls konservativere Abtreibungsregelungen zu implementieren.

In repräsentativ organisierten Demokratien kommt es jedoch nicht nur darauf an, welche Positionen politische Akteure im Vorfeld einer Wahl versprechen, sondern auch, ob und - wenn ja - in welchem Umfang diese Präferenzen auch umgesetzt und implementiert werden (Powell 2000, 2004). Sollte Letzteres nicht oder nur zu einem geringen Grad der Fall sein, so riskieren die regierenden Parteien, bei der nächsten Wahl Stimmenverluste zu erleiden. Somit ist - mit Rückgriff auf die Literatur zur Parteiendifferenzthese (vgl. Hibbs 1977; Schmidt 1996; Bräuninger 2005; Cusack u. Beramendi 2006) - zu erwarten, dass mit zunehmender Stärke gesellschaftspolitisch progressiv ausgerichteter Parteien in der Legislative ein entsprechender Politikwandel stattfinden wird. Das für unsere Untersuchung einschlägige Entscheidungsgremium ist der Kreistag bzw. im Fall der kreisfreien Städte der Gemeinderat, da diese Änderungen der Gebührenordnung zustimmen müssen. Somit erwarten wir, dass ein Kreistag oder ein Gemeinderat mit einer zunehmenden Vertretung von gesellschaftspolitisch progressiv ausgerichteten Parteien die Gebührenhöhe für eingetragene Lebenspartnerschaften niedriger ansetzen sollte.

Eine solche Perspektive würde jedoch die Rolle der Kreis- bzw. Stadtverwaltung unterschätzen, da sie es ist, die einen Vorschlag für eine Gebühr ausarbeitet und dem Parlament in Form einer Änderung der Gebührenordnung vorlegt (vgl. etwa Wegrich 2006). Hieraus folgt, dass die Verwaltungen der Landkreise und kreisfreien Städte als „Agenda-Setzer“ hinsichtlich der Höhe des Gebührensatzes fungieren können (vgl. Schnapp 2004). Der Vorschlag über die Höhe einer Gebühr legt somit zu einem maßgeblichen Grad fest, wie hoch der veranschlagte Betrag sein wird. Der ökonomischen Theorie der Bürokratie zufolge verfolgt die Verwaltung eigene, ihren Nutzen maximierende Interessen, baut jedoch insbesondere auf den individuellen Präferenzen der Behördenleiter auf (Niskanen 1971; vgl. Shepsle u. Bonchek 1997, S. 348-353; Mueller 2003, S. 362-380; Holzinger 2009, S. 548-549).

Dies bedeutet, dass die Verwaltung die Präferenzen in der Legislative - und damit der Kreistage und der Gemeinderäte in den kreisfreien Städten - nutzen kann, um überdurchschnittlich hohe Gebühren zu verlangen und damit ihre Einnahmen zu steigern. So sollte ein gesellschaftspolitisch konservativ dominiertes Parlament eine eher geringe Anzahl von gleichgeschlechtlichen Lebenspartnerschaften in der jeweiligen regionalen Einheit präferieren. Um dieses Ergebnis zu erreichen, sollte - aus Perspektive der politischen Steuerung (vgl. etwa Scharpf 1989; Mayntz 1990; Lütz 2002; Mayntz u. Scharpf 2005) - ein möglichst hoher Gebührensatz von der Verwaltung vorgeschlagen werden. ${ }^{2}$ Aus dieser, die Rolle

2 Aufgrund der eher geringen Anzahl von Lebenspartnerschaften ist der finanzielle „Gewinn“, den die Verwaltung aus den überhöhten Gebühren machen könnte, natürlich eher gering. Dennoch bleibt der theoretische Mechanismus gleich: Wenn die Chance zu einer Maximierung des Budgets, auch wenn sie noch so gering ausfallen mag, vorhanden ist, dann sollte die Bürokratie in Form der Behördenleiter diese auch ergreifen. 
der Verwaltung im Agendasetzungsprozess betonenden Perspektive wie auch auf der Grundlage der Parteiendifferenztheorie lässt sich die Erwartung ableiten, dass die Gebührenhöhe für eingetragene Lebenspartnerschaften über die gesellschaftspolitischen Präferenzen der Parteien und Abgeordneten im Parlament determiniert ist. Aus diesen Überlegungen leiten wir unsere erste Hypothese ab:

H1: Je höher der Anteil gesellschaftspolitisch progressiver Parteien in einem Kreistag oder Gemeinderat in Baden-Württemberg ist, desto geringer sollten die Mehrkosten für eingetragene Lebenspartnerschaften im Vergleich zur Eheschließung ausfallen.

Neben dieser Hypothese lassen sich mit Rückgriff auf Erwägungen hinsichtlich der politischen Kultur, des ökonomischen Problemdrucks, dem sich ein Landkreis oder eine kreisfreie Stadt gegenübergestellt sieht, und des Konzepts des „PolicyLernens" weitere Erwartungen bezüglich der Gebührenhöhe für eingetragene Lebenspartnerschaften ableiten. So deuten die Ergebnisse von Studien zur politischen Kultur in den Regionen Deutschlands darauf hin, dass sowohl eher kurzfristig orientierte Faktoren wie Wertepräferenzen und Wahlverhalten der Bevölkerung als auch das Bestehen einer langfristig wirkenden „politischen Deutungskultur“ nicht nur zwischen den - zum Teil nach dem Zweiten Weltkrieg künstlich geschaffenen - Bundesländern, sondern auch zwischen den Regionen innerhalb der Länder variieren (vgl. etwa Oberndörfer u. Schmitt 1991; Rohe 1992, 1996; Pickel u. Pickel 2006, S. 123-128; Wehling 2006; Völkl et al. 2008, S. 12-13; Magin et al. 2009; Müller 2009; Bräuninger u. Debus 2012).

Mit Hinblick auf gesellschaftspolitische Fragestellungen sollte insbesondere in ländlich und kirchennah geprägten Regionen eine überdurchschnittlich hohe Ablehnung gegenüber Einrichtungen wie Lebenspartnerschaften, die dem traditionellen Familienbild widersprechen, zu erwarten sein, sodass in solchen Landkreisen die Gebühren für eingetragene Lebenspartnerschaften tendenziell höher als für Eheschließungen ausfallen sollten. Dieser Umstand könnte für die Festlegung der Gebührenhöhe in zweierlei Hinsicht relevant sein. Zum einen ist es wahrscheinlich, dass Mitglieder der Verwaltung eines stärker urbanisierten und weniger religiös geprägten Landkreises - auch aufgrund ihrer eigenen Sozialisierung - eine geringere Ablehnung gegenüber alternativen Lebensweisen hegen und somit niedrigere Gebühren präferieren. Komplementär sollte sich die Situation in ländlichen und stärker religiös geprägten Landkreisen in der Form darstellen, dass die Mitglieder der Verwaltung aufgrund des Symbolcharakters einen höheren Gebührensatz bevorzugen. Zum anderen ist zu erwarten, dass sich die politischen Akteure an der politischen Kultur in den Regionen orientieren und ihre Position im Vergleich zur offiziellen Parteilinie je nach Region konservativer oder moderater sein wird, um den Wählern entsprechende „ideologische Signale“ (Pappi u. Shikano 2004) zu senden und somit ihre Wiederwahlchancen zu erhöhen. Die zweite hier zu testende Hypothese lautet daher:

H2: Je stärker urbanisiert und je weniger religiös ein Landkreis bzw. eine kreisfreie Stadt geprägt sind, desto geringer sollten die Mehrkosten für das Schließen einer eingetragenen Lebenspartnerschaft im Vergleich zu einer Ehe ausfallen. 
Wie bereits aufgezeigt wurde können Gebühren jeglicher Art jedoch auch ein Instrument der Exekutive darstellen, um die finanzielle Lage einer Gebietskörperschaft zu verbessern. So hat beispielsweise die Stadt Rottweil aufgrund der Wirtschafts- und Finanzkrise Beschlüsse auf den Weg gebracht, die zum Zweck der Haushaltskonsolidierung eine Erhöhung von Steuern und Gebühren vorsehen (Stadtnachrichten Rottweil 25.11.2010). Dabei sollte es irrelevant sein, ob es sich bei der Leistung, für die eine Gebühr verlangt wird, um ein wirtschafts- und gesellschaftspolitisch konfliktäres Issue handelt oder nicht. Politische Akteure, die das Ziel der Wiederwahl verfolgen (Downs 1957; Strøm u. Müller 1999), sollten die Einführung hoher Gebühren bzw. die Erhöhung bereits bestehender Abgabensätze aufgrund des negativen Effekts auf die Wiederwahlchancen vermeiden. Somit müssen externe Bedingungen in Form eines ökonomischen Problemdrucks gegeben sein, die politische Akteure dazu bewegen, höhere Gebühren zu verlangen (vgl. etwa Wagschal u. Wenzelburger 2009). Im Hinblick auf die Höhe von Steuern und Gebühren auf kommunaler und regionaler Ebene sollte vor allem eine schlechte wirtschaftliche Lage dazu führen, dass höhere Gebühren auch im Fall eingetragener Lebenspartnerschaften verlangt werden. Hieraus leiten wir die dritte Hypothese ab:

H3: Je höher die Verschuldung und die Arbeitslosigkeit einer kreisfreien Stadt bzw. eines Landkreises sind, desto höher sollten die Mehrkosten für das Schließen einer eingetragenen Lebenspartnerschaft im Vergleich zu einer Ehe ausfallen.

Weitere theoretisch relevante Ansätze spiegeln Aspekte des Policy-Lernens wider (vgl. etwa Sabatier 1993; Hall 1993; Bandelow 2003; Biegelbauer 2007; Knill et al. 2007). In den Phasen des Policy-Zyklus, in denen es um die Definition eines Problems und die konkrete Ausgestaltung eines Politikinhalts geht, können sich die Akteure gerade im Fall einer neuen Policy an den Handlungsweisen der politischen Entscheidungsträger in räumlich benachbarten Gebietskörperschaften orientieren (vgl. Bogumil u. Jann 2009, S. 24-25).

Es erscheint daher plausibel anzunehmen, dass sich sowohl die Entscheidungsträger als auch die Kreis- und Stadtverwaltungen an der Höhe der Gebühren für die Schließung einer eingetragenen Lebenspartnerschaft in den benachbarten Regionen orientieren, wenn sie einen Gebührenvorschlag machen bzw. über diesen entscheiden. Diese Überlegung basiert auf der Annahme, dass zwischen benachbarten Kreisen und Städten generell von einem hohen Ausmaß der Interaktion der jeweiligen Entscheidungsträger ausgegangen werden kann. Dies erleichtert potenziell die Übernahme von Konzepten, die in Nachbarkommunen bereits entwickelt wurden, insbesondere dann, wenn auf diese Weise Kosten der eigenen Entscheidungsfindung reduziert werden können oder ein gewisser Zeitdruck für die Umsetzung bestimmter Maßnahmen besteht (vgl. Bennett 1991, S. 223; Sommerer 2010). Unsere vierte Hypothese lautet daher:

H4: Die Höhe der Gebühren für die Schließung eingetragener Lebenspartnerschaften in einem Landkreis oder einer kreisfreien Stadt hängt von der Gebührenhöhe in den jeweils benachbarten Kreisen und kreisfreien Städten ab. 


\section{Daten und Operationalisierung}

Zentral für die Beantwortung der hier aufgeworfenen Fragestellung ist die Ermittlung der Höhe der Gebühren, die bei der Schließung einer eingetragenen Lebenspartnerschaft in einem baden-württembergischen Landkreis oder einer kreisfreien Stadt anfallen. Zum anderen müssen - im Fall der erklärenden Variablen - solche Faktoren ermittelt werden, die die politisch-kulturellen, sozialstrukturellen und makroökonomischen Eigenschaften sowie die Stärke der gesellschaftspolitisch progressiven Akteure in den Parlamenten der baden-württembergischen Kreise und kreisfreien Städte wiedergeben. Informationen hinsichtlich der Ausprägungen der abhängigen Variablen sind meist über die Internetauftritte der jeweiligen Kreise und Städte erhältlich. In den verbleibenden Fällen wurden die Gebührensätze über telefonische Anfragen bei den Kreis- und Stadtverwaltungen erfragt. ${ }^{3}$ Die abhängige Variable unserer Untersuchung stellt die Differenz zwischen der Gebührenhöhe für eingetragene Lebenspartnerschaften zum Zeitpunkt der Ermittlung im Oktober und November 2010 und den tatsächlichen Kosten dar. Als Näherungsindikator für die tatsächlichen Kosten ziehen wir den Gebührensatz für die Schließung einer Ehe heran, der zum Zeitpunkt dieser Untersuchung in Baden-Württemberg bei $40 €$ liegt. ${ }^{4}$

Für die Ermittlung der Stärke der politischen Akteure auf kommunaler Ebene mit gesellschaftspolitisch progressiven Präferenzen werden die Sitzanteile der Parteien in den 2009 neu gewählten Kreistagen und - im Fall der kreisfreien Städte - Gemeinderäten herangezogen. Auf der Grundlage von Inhaltsanalysen der zwischen 2001 und 2011 verfassten Landtagswahlprogramme können Sozialdemokraten, Freidemokraten, Bündnis 90/Die Grünen sowie die Linke als politische Akteure mit gesellschaftspolitisch progressiven Positionen bestimmt werden (Bräuninger u. Debus 2008, 2012). Hingegen ist die baden-württembergische $\mathrm{CDU}$ in dieser Politikdimension eher konservativ ausgerichtet. Abbildung $1 \mathrm{gibt}$ die ideologische Parteienkonstellation Baden-Württembergs für den oben angegebenen Zeitraum wieder. Es wird deutlich, dass FDP und Union zwar ähnliche wirtschafts- und sozialpolitische Positionen vertreten, jedoch in der Innen-, Rechts- und Gesellschaftspolitik Liberale, Grüne, die Linke und auch die Sozialdemokraten im Gegensatz zur CDU moderate bis explizit progressive Haltungen in ihren Landtagswahlprogrammen einnehmen. Die unterschiedliche Position von Union und Freidemokraten in gesellschaftspolitischen Fragen zeigte sich zudem bei namentlichen Abstimmungen im Landtag von Baden-Württemberg (vgl. etwa Stecker 2011): So wichen vier Abgeordnete der baden-württembergischen Liberalen bei der Abstimmung über die Reform des öffentlichen Dienstrechts, die keine Gleichstellung gleichgeschlechtlicher Lebenspartnerschaften mit der Ehe vorsah,

3 In diesem Zusammenhang möchten wir uns bei Christian Rubba und Alexander Dietrich für die hervorragende Unterstützung bei der Datenerhebung und der Erstellung des Datensatzes bedanken. Zudem bedanken wir uns bei Stephan Grohs und Simon Munzert sowie der PVS-Redaktion und den beiden anonymen Gutachtern für hilfreiche Anmerkungen und Verbesserungsvorschläge.

4 Im Enzkreis sowie in den Landkreisen Heilbronn und Konstanz bestehen keine fixen Gebührensätze, sondern die Höhe der Gebühren wird pro angefangener Stunde (Enzkreis und Kreis Heilbronn) bzw. je angefangener Viertelstunde (Konstanz) berechnet. Im Folgenden wird von einer Stunde als Gesamtdauer der Verpartnerung ausgegangen. 
im Oktober 2010 von der Linie der CDU/FDP-Koalition aufgrund der ihrer Ansicht nach diskriminierenden Gesetzesvorlage der Landesregierung ab (vgl. Landtag von Baden-Württemberg 2010, S. 7263).

Abbildung 1: Wirtschafts- und gesellschaftspolitische Positionen der badenwürttembergischen Parteien zu den Landtagswahlen von 2001 bis 2011

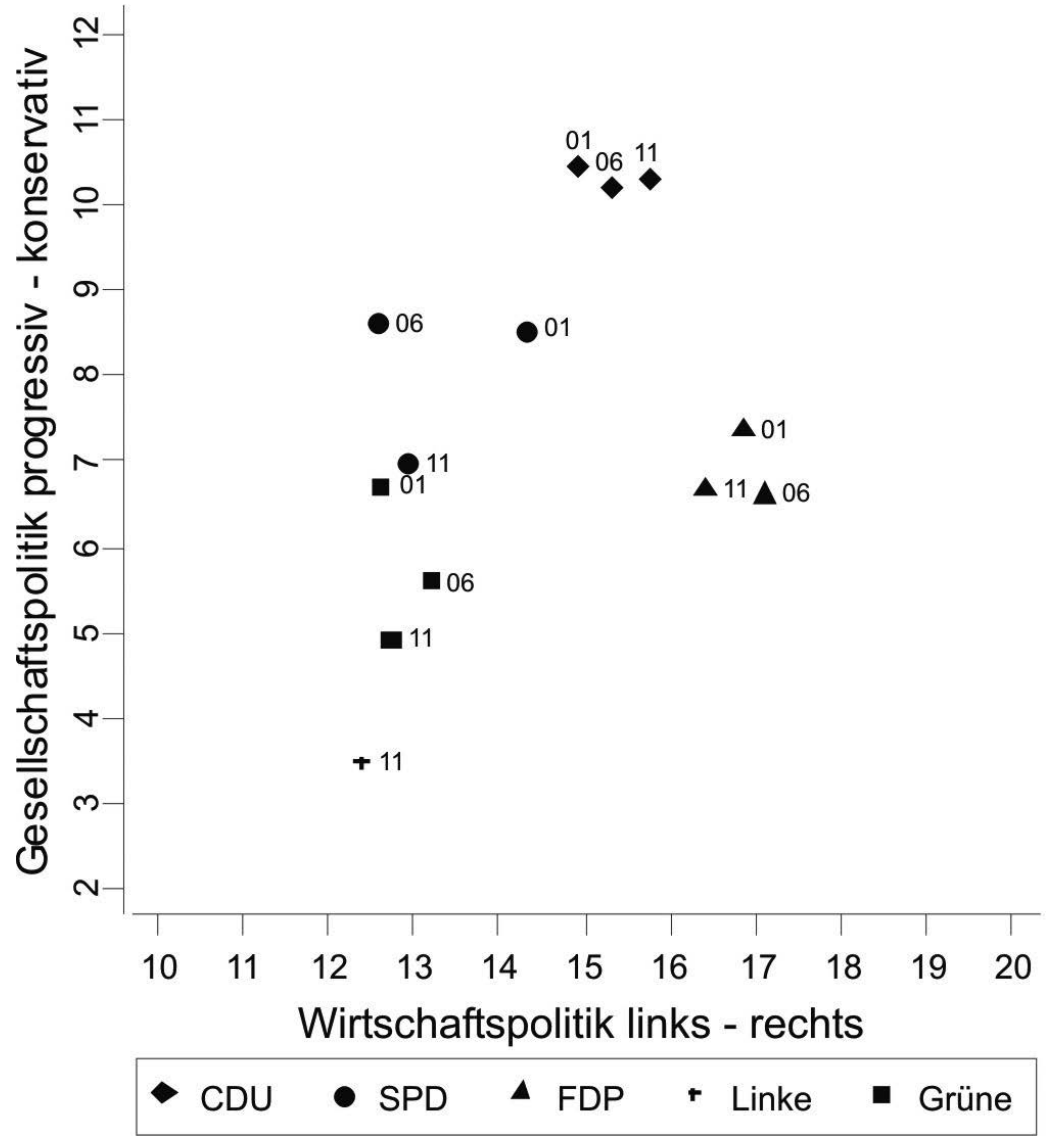

Quelle: Bräuninger u. Debus 2008, 2012.

Für die im Südwesten traditionell auf kommunaler Ebene sehr starken Freien Wähler liegen keine Daten hinsichtlich ihrer gesellschaftspolitischen Position vor, da sie bei Landtagswahlen nicht antreten und daher kein Wahlprogramm formulieren. Studien, die das programmatische Profil solcher Wählergemeinschaften untersuchen, kommen jedoch zu dem Ergebnis, dass diese inhaltlich eher den Christdemokraten - auch aufgrund ihrer in Baden-Württemberg hauptsächlich aus dem Milieu des ,alten Mittelstands' rekrutierten Mitglieder - nahe stehen und damit gesellschaftspolitisch eher konservativ ausgerichtet sind (vgl. Holtmann 2002, 
S. 423-426; Holtmann 2008, S. 8; Reiser u. Krappidel 2008, S. 85). Um zu identifizieren, welcher Effekt von der Stärke der Freien Wähler als auch der übrigen Parteien - CDU, SPD, FDP, Grünen und der Linken - in den Kommunalparlamenten ausgeht, gehen in gesonderten Regressionsmodellen die Sitzanteile dieser Parteien separat anstelle der aggregierten Variable „Stärke der gesellschaftspolitisch progressiv ausgerichteten Parteien " ein. Zusätzlich wird eine Variable in die Analyse eingebaut, die Aufschluss über die Zugehörigkeit des jeweiligen Landrates oder Oberbürgermeisters zu einer gesellschaftspolitisch progressiven Partei - also SPD, Bündnis 90/Die Grünen, FDP oder Linke - gibt.

Informationen zur Anzahl der Parlamentssitze der Parteien, den sozialstrukturellen Eigenschaften der baden-württembergischen Kreise und kreisfreien Städte sowie deren ökonomischem Problemdruck sind weitgehend über das statistische Landesamt Baden-Württemberg (www.statistik.baden-wuerttemberg.de) sowie über die Regionaldatenbank Deutschland (www.regionalstatistik.de) abrufbar. Zur Operationalisierung des ökonomischen Problemdrucks, der zu generell höheren Gebühren führen sollte, werden die Verschuldung je Einwohner eines Landkreises bzw. einer kreisfreien Stadt sowie die Arbeitslosenquote zum Stand Jahresende 2008 herangezogen.

Schwieriger gestaltet sich die Umsetzung politisch-kultureller Faktoren in numerische Variablen. Während der Grad der Urbanisierung über den Anteil der Beschäftigten im primären Sektor bestimmt werden kann, so ist die Messung der Religiosität auf kommunaler Ebene schwierig. Informationen zur konfessionellen Zusammensetzung der Bevölkerung je Land- und Stadtkreis auf der Grundlage von Daten des Statistischen Landesamtes Baden-Württemberg reichen nur bis Dezember 1995 (vgl. Statistisches Landesamt Baden-Württemberg 1997). Eine Möglichkeit zur Generierung aktuellerer Daten bieten die Umfragen im Rahmen des „Forsa-Bus“, die wöchentlich durchgeführt werden. Dadurch ergibt sich eine hohe Fallzahl, die es zulässt, Analysen auch auf Landkreisebene durchzuführen. Wir verwenden hier die Daten des Forsa-Bus 2009 (ZA-Nr. 5049), um den Anteil der Befragten pro Landkreis und kreisfreier Stadt zu ermitteln, die sich als konfessionslos bezeichnen. ${ }^{5}$

Der Aspekt des „Policy-Lernens“ wird durch einen räumlichen Schätzer abgedeckt, der den ungewichteten mittleren Gebührensatz für eingetragene Lebenspartnerschaften in den jeweils an einen Landkreis oder eine kreisfreie Stadt angrenzenden Gebietskörperschaften wiedergibt. ${ }^{6}$ Zudem wird eine Variable in die Analyse aufgenommen, die die kreisfreien Städte kennzeichnet. Dies geschieht vor allem deswegen, da in Baden-Württemberg die kreisfreien Städte auch die Eigenschaften von Gemeinden haben und somit - im Gegensatz zu den Landkreisen - auf einen gemeinsamen Verwaltungsapparat zurückgreifen können, sodass eine

5 Der Datensatz des Forsa-Bus 2009 wurde dankenswerterweise über das Archiv der GESIS verfügbar gemacht.

6 Im Fall von Land- und Stadtkreisen, die an ein anderes Bundesland grenzen, wird auch die dort für das Schließen einer Lebenspartnerschaft fällige Gebühr berücksichtigt. Diese beträgt in Rheinland-Pfalz und Hessen $40 €$ und in Bayern $50 €$ (vgl. http://www.standesbeamte.de/standesbeamte_gebuehren.html; Zugriff am 22. Juli 2011). 
Veranschlagung hoher Mehrkosten für die Schließung eingetragener Lebenspartnerschaften generell unwahrscheinlicher erscheint (vgl. etwa Burgi 2010, S. 306).

Die hier angewandte Methode zur Bestimmung der ausschlaggebenden Faktoren für Mehrkosten eingetragener Lebenspartnerschaften im Vergleich zu den Gebühren, die bei einer Eheschließung anfallen, ist eine Tobit-Regression, da die Mehrkosten nach unten begrenzt sind und nicht unter den Betrag von $0 €$ fallen können. Die Gebührenmehrkosten in einem Landkreis oder kreisfreien Stadt $i$ sollten von den oben beschriebenen und in der folgenden Gleichung aufgeführten Faktoren abhängen:

Mebrkosten $_{i}=\beta_{0}+\beta_{1} \times$ PROGRESSIV $+\beta_{2} \times$ KONFESSIONSLOS + $\beta_{3} \times$ BEVÖLKERUNGSDICHTE $+\beta_{4} \times$ PRIMÄRER SEKTOR $+\beta_{5} \times$ VER $S C H U L D U N G+\beta_{6} \times$ ARBEITSLOSENQUOTE $+\beta_{7} \times$ GEBÜHRENHÖHE $N A C H B A R K R E I S E+\beta_{8} \times S T A D T K R E I S+\varepsilon_{i}$

\section{Analyse}

Bevor die Ergebnisse der Tobit-Regressionsanalysen und damit der Test der oben aufgeführten Hypothesen präsentiert werden, soll im folgenden Unterabschnitt zunächst auf die Ausprägungen der abhängigen und unabhängigen Variablen sowie insbesondere auf das Ausmaß der Unterschiede in den Mehrkosten für die Schließung eingetragener Lebenspartnerschaften zwischen den Landkreisen und kreisfreien Städten Baden-Württembergs eingegangen werden.

\subsection{Beschreibung der Daten}

Tabelle 1 gibt eine Übersicht über die Ausprägungen aller hier herangezogenen abhängigen und unabhängigen Variablen sowie der gemäß den Hypothesen erwarteten Richtung des Einflusses der erklärenden Faktoren. Mit Hinblick auf die abhängige Variable wird deutlich, dass die Mehrkosten, die ein gleichgeschlechtliches Paar im Vergleich zu einem heterosexuellen Paar bei der Verpartnerung in Baden-Württemberg zahlen muss, nicht nur im Durchschnitt knapp $66 €$ betragen, sondern auch deutlich zwischen den Land- und Stadtkreisen variieren: Während in manchen Kreisen und Städten keine Mehrkosten bestehen, so liegt das Maximum des Kostenunterschiedes bei $198 €$. 
Tabelle 1: Deskription der abhängigen und unabhängigen Variablen

\begin{tabular}{|c|c|c|c|c|c|}
\hline Variable & $\begin{array}{l}\text { Mittel- } \\
\text { wert }\end{array}$ & SD & Min. & Max. & $\begin{array}{c}\text { Erwar- } \\
\text { teter } \\
\text { Effekt }\end{array}$ \\
\hline \multicolumn{6}{|l|}{ Abhängige Variable } \\
\hline Gebührenmehrkosten & 65,96 & 49,60 & 0 & 198 & \\
\hline \multicolumn{6}{|l|}{ Unabhängige Variablen } \\
\hline \multicolumn{6}{|l|}{ Präferenzen politischer Akteure } \\
\hline Sitzanteil progressiver Parteien ( $\%)$ & 40,97 & 10,30 & 18,75 & 64,58 & - \\
\hline Sitzanteil CDU (\%) & 35,25 & 8,00 & 20,83 & 56,25 & + \\
\hline Sitzanteil SPD (\%) & 18,10 & 4,98 & 8,33 & 33,33 & - \\
\hline Sitzanteil FDP (\%) & 9,05 & 4,35 & 0 & 24 & - \\
\hline Sitzanteil Bündnis 90/Die Grünen (\%) & 12,32 & 5,25 & 4,08 & 29,17 & - \\
\hline Sitzanteil Freie Wähler (\%) & 22,48 & 7,83 & 0 & 38,46 & + \\
\hline Sitzanteil Linke (\%) & 1,50 & 1,82 & 0 & 8,33 & - \\
\hline Sitzanteil Sonstige (\%) & 1,30 & 2,05 & 0 & 8,33 & \\
\hline Progressiver Landrat/Oberbürgermeister & 0,11 & 0,32 & 0 & 1 & - \\
\hline \multicolumn{6}{|l|}{ Politische Kultur } \\
\hline Anteil der Konfessionslosen (\%) & 20,78 & 5,30 & 10,38 & 31,76 & + \\
\hline $\begin{array}{l}\text { Anteil Beschäftigter im primären } \\
\text { Sektor }(\%)\end{array}$ & 2,09 & 1,29 & 0,23 & 5,32 & + \\
\hline \multicolumn{6}{|l|}{ Problemdruck } \\
\hline Verschuldung pro Kopf $(€)$ & 589,10 & 242 & 209,20 & 1477,03 & + \\
\hline Arbeitslosenquote $(\%)$ & 4,22 & 1,20 & 2,30 & 7,50 & + \\
\hline \multicolumn{6}{|l|}{ „Policy-Lernen“ } \\
\hline Gebührenhöhe benachbarter Kreise/Städte & 101,60 & 31,11 & 43 & 157,25 & + \\
\hline Kreisfreie Stadt & 0,18 & 0,39 & 0 & 1 & - \\
\hline
\end{tabular}

Quelle: www.statistik.baden-wuerttemberg.de, www.regionalstatistik.de, Daten des Forsa-Bus von 2009 (ZA-Nr. 5049) sowie eigene Erhebungen. Die Anzahl der Fälle beträgt jeweils N=44.

Abbildung 2 zeigt anhand einer schematischen Karte Baden-Württembergs auf, wo keine Mehrkosten anfallen bzw. wo diese gering, hoch oder sehr hoch sind. In vier Stadtkreisen - Heidelberg, Karlsruhe, Stuttgart und Ulm - werden mit Stand von November 2010 keine Unterschiede zwischen Ehe und Lebenspartnerschaft hinsichtlich der Gebührenhöhe gemacht. In den anderen Kreisen und kreisfreien Städten werden - wiederum zum Zeitpunkt der Erhebung - hingegen deutlich höhere Gebühren als für eine Eheschließung verlangt. Das Maximum von $198 €$ findet sich im Zollernalbkreis. Ebenfalls sehr hoch fallen die Mehrkosten im Landkreis Sigmaringen $(160 €)$ sowie im Bodenseekreis $(150 €)$ aus. $^{7}$

7 Weder die Anzahl der geschlossenen Lebenspartnerschaften noch der Anteil, den die aus dem Schließen von Lebenspartnerschaften resultierenden Gebühreneinnahmen an allen Gebühreneinnahmen eines Landkreises oder einer kreisfreien Stadt ausmachen, liegen laut Auskunft des Statistischen Landesamts Baden-Württemberg vor und können daher nicht in der Analyse berücksichtigt werden. 
Abbildung 2: Gebührenmehrkosten für eingetragene Lebenspartnerschaften im Vergleich zur Eheschließung in den Land- und Stadtkreisen Baden-Württembergs

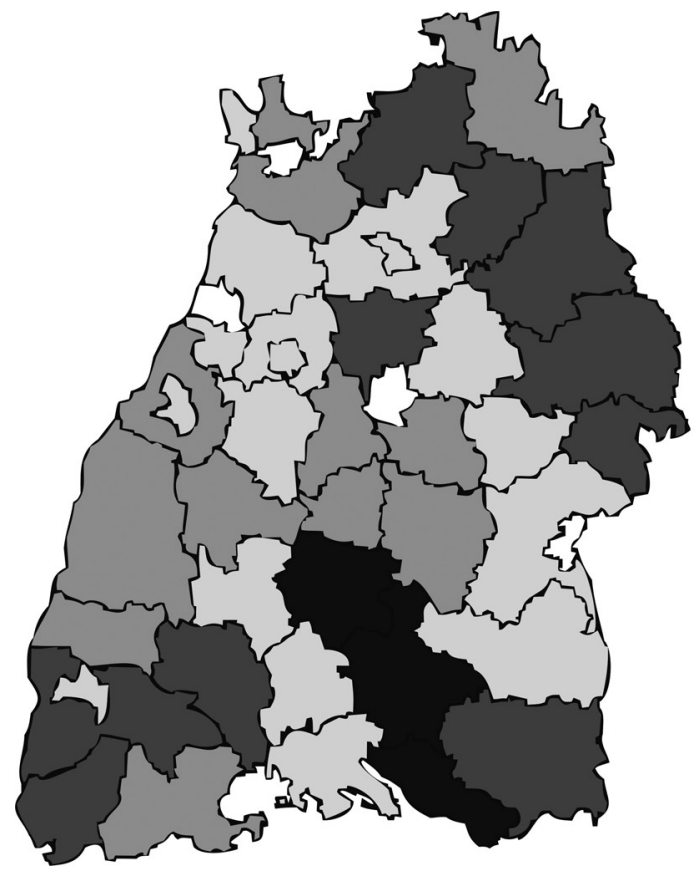

Keine Mehrkosten

Mehrkosten von $1 €$ bis $49 €$

Mehrkosten von $50 €$ bis $99 €$

Mehrkosten von $100 €$ bis $149 €$

Mehrkosten von $150 €$ bis $198 €$

Quelle: Eigene Darstellung.

\subsection{Multivariates Analysemodell}

Welche Faktoren beeinflussen die Varianz in der Gebührenhöhe für das Schließen eingetragener Lebenspartnerschaften in Baden-Württemberg? Die in Tabelle 2 abgetragenen Ergebnisse der Regressionsanalyse bestätigen den in der ersten Hypothese vermuteten negativen Zusammenhang zwischen dem Sitzanteil gesellschaftspolitisch progressiver Parteien und den Mehrkosten für die Schließung eingetragener Lebenspartnerschaften: Je höher der Anteil für SPD, FDP, Grüne und Linke in einem Kreistag oder - im Fall der kreisfreien Städte - einem Gemeinderat ist, desto niedriger fallen - unter Kontrolle aller weiteren, theoretisch hergeleiteten Variablen - die Mehrkosten aus. Somit kann die erste Hypothese bestätigt werden.

Zieht man zur Interpretation der Analyseergebnisse die ebenfalls in Tabelle 2 ausgewiesenen marginalen Effekte der einzelnen Variablen heran, so würden mit dem Anwachsen des Sitzanteils gesellschaftspolitisch progressiver Parteien um eine Einheit in denjenigen Kreisen und kreisfreien Städten, die einen „Gebührenaufschlag “ vornehmen, die Mehrkosten um den Betrag von 1,70€ sinken. Ein substanziell gesehen stärkerer Effekt geht von der Höhe der Arbeitslosenquote 
und dem Gegensatz zwischen Landkreis und kreisfreier Stadt aus. So steigen die Gebühren - wiederum dort, wo sie über denjenigen für die Schließung einer Ehe liegen - gemäß dem ersten Modell um 28,49 €, wenn die Arbeitslosenquote um eine Einheit ansteigt. Im Vergleich zu den baden-württembergischen Landkreisen sind die auf den „eigentlichen“ Gebührensatz von $40 €$ aufgeschlagenen Mehrkosten in den kreisfreien Städten um durchschnittlich $67 €$ niedriger.

Wird anstelle der aggregierten Variable nach der Stärke der einzelnen Parteien im jeweiligen Kommunalparlament differenziert ${ }^{8}$, dann ergibt sich für die Sitzanteile von FDP und Grünen ein statistisch signifikanter und negativer Effekt: Je stärker Liberale und Grüne in einem Kreistag oder im Gemeinderat einer kreisfreien Stadt in Baden-Württemberg vertreten sind, desto niedriger sind die Mehrkosten, die die Verwaltung auf die Gebühren zur Schließung einer Ehe aufgeschlagen hat. Eine zunehmende Stärke der Freien Wähler in den kommunalen Parlamenten hat keinen Einfluss auf die Mehrkosten, was die Vermutung stärkt, dass die Freien Wählervereinigungen zumindest in Baden-Württemberg gesellschaftspolitisch nicht progressiv ausgerichtet sind. Dieses Ergebnis deckt sich mit den bisherigen Befunden aus der Forschungsliteratur (vgl. Holtmann 2002, 2008; Reiser u. Krappidel 2008). Die in Modellen 3 und 4 zusätzlich eingebaute Variable der Zugehörigkeit des Landrats oder Oberbürgermeisters zu einer gesellschaftspolitisch progressiv ausgerichteten Partei hat zwar den erwarteten negativen Effekt, ist jedoch auf den gängigen Signifikanzniveaus nicht signifikant von Null verschieden. Mit zunehmender Sitzstärke von FDP bzw. Grünen in den Kreistagen und Gemeinderäten der kreisfreien Städte, die einen „Gebührenaufschlag“ aufweisen, würden - gemäß Model 4 - die Mehrkosten für das Schließen einer Lebenspartnerschaft um 4,80€ bzw. 5,48 € sinken.

Zudem weisen die Modelle statistisch signifikante Evidenz für die dritte Hypothese auf: So steigt die Gebührenhöhe für eingetragene Lebenspartnerschaften, wenn ökonomischer Problemdruck, dem sich ein Kreis oder eine kreisfreie Stadt gegenübersieht, in Form von insbesondere hoher Arbeitslosigkeit besteht. Gemischt sind die Resultate hingegen im Falle der Variablen, die politisch-kulturelle Faktoren widerspiegeln. Berücksichtigt man nur Modelle 2 und 4, so steigen wie erwartet nicht nur die Mehrkosten an, je höher der Anteil der Beschäftigten im primären Wirtschaftssektor ist. Vielmehr sinkt die Höhe der Gebühren zur SchlieBung einer Lebenspartnerschaft auch mit einem Anwachsen des Anteils der Konfessionslosen in einem Landkreis oder einer kreisfreien Stadt. Die Effekte dieser beiden Variablen weisen in Modellen 1 und 3 zwar noch dieselbe Richtung auf, sind jedoch nicht mehr statistisch signifikant. ${ }^{9}$ Wie die geschätzten marginalen Effekte deutlich machen, geht der stärkste Effekt auf die Mehrkosten auch in den Modellen 2 und 4 von der Höhe der Arbeitslosigkeit als auch vom Anteil der Beschäftigten im primären Sektor aus. Zudem würde in statistisch signifikanter Wei-

8 Wir berücksichtigen lediglich CDU, SPD, FDP, Bündnis 90/Die Grünen, Die Linke und Freie Wähler als die zentralen politischen Parteien. Alle übrigen Parteien, die in einem Kreistag oder Stadtparlament vertreten sind, gehen als "Sonstige“ in die Analyse ein und bilden die Referenzkategorie in den Regressionsmodellen 2 und 4.

9 Die Regressionsdiagnostik weist keine größeren Probleme beim Schätzen der Modelle auf. 
se der Aufschlag auf den Gebührensatz sinken (um rund 3,50 €), wenn in Landkreisen und kreisfreien Städten mit „Gebührenaufschlägen“ der Anteil der Konfessionslosen um eine Einheit anwächst.

Für die vierte Hypothese, die von einem Einfluss der Gebührenhöhe in den Bundesländern, Kreisen oder kreisfreien Städten ausging, die jeweils an einen Land- oder Stadtkreis angrenzen, ergibt sich keine Bestätigung. Hingegen sind die Gebührenmehrkosten in den Stadtkreisen Baden-Württembergs signifikant niedriger als in den Landkreisen, was bereits die deskriptive Analyse anhand von Abbildung 2 verdeutlichen konnte. Insgesamt betrachtet können wir auf der Grundlage der in Tabelle 2 präsentierten Regressionsergebnisse die Hypothesen 1 und 3 bestätigen. Die Analysen unterstützen zu einem gewissen Grad auch die zweite, politische-kulturelle Faktoren hervorhebende Erwartung. Wir können somit festhalten, dass - im Fall der baden-württembergischen Sonderregelung der Handhabung eingetragener Lebenspartnerschaften - die Stärke gesellschaftspolitisch progressiver Parteien auf kommunaler Ebene (und hier insbesondere die Sitzanteile von FDP und Bündnis 90/Die Grünen) unter Kontrolle weiterer Faktoren einen negativen Effekt auf die Gebührenhöhe aufweisen. Dieser Effekt ist zwar nicht so stark ausgeprägt wie derjenige, der von Problemdruckvariablen wie dem Grad der regionalen Arbeitslosigkeit oder der spezifischen politischen Kultur einer Region - gemessen anhand des Anteils der im landwirtschaftlichen Bereich beschäftigten Personen - ausgeht, jedoch auf gängigen statistischen Testniveaus signifikant. Somit spielen parteipolitische Präferenzen auch auf lokaler und regionaler Ebene eine zentrale Rolle für die Ergebnisse des politischen Prozesses. 


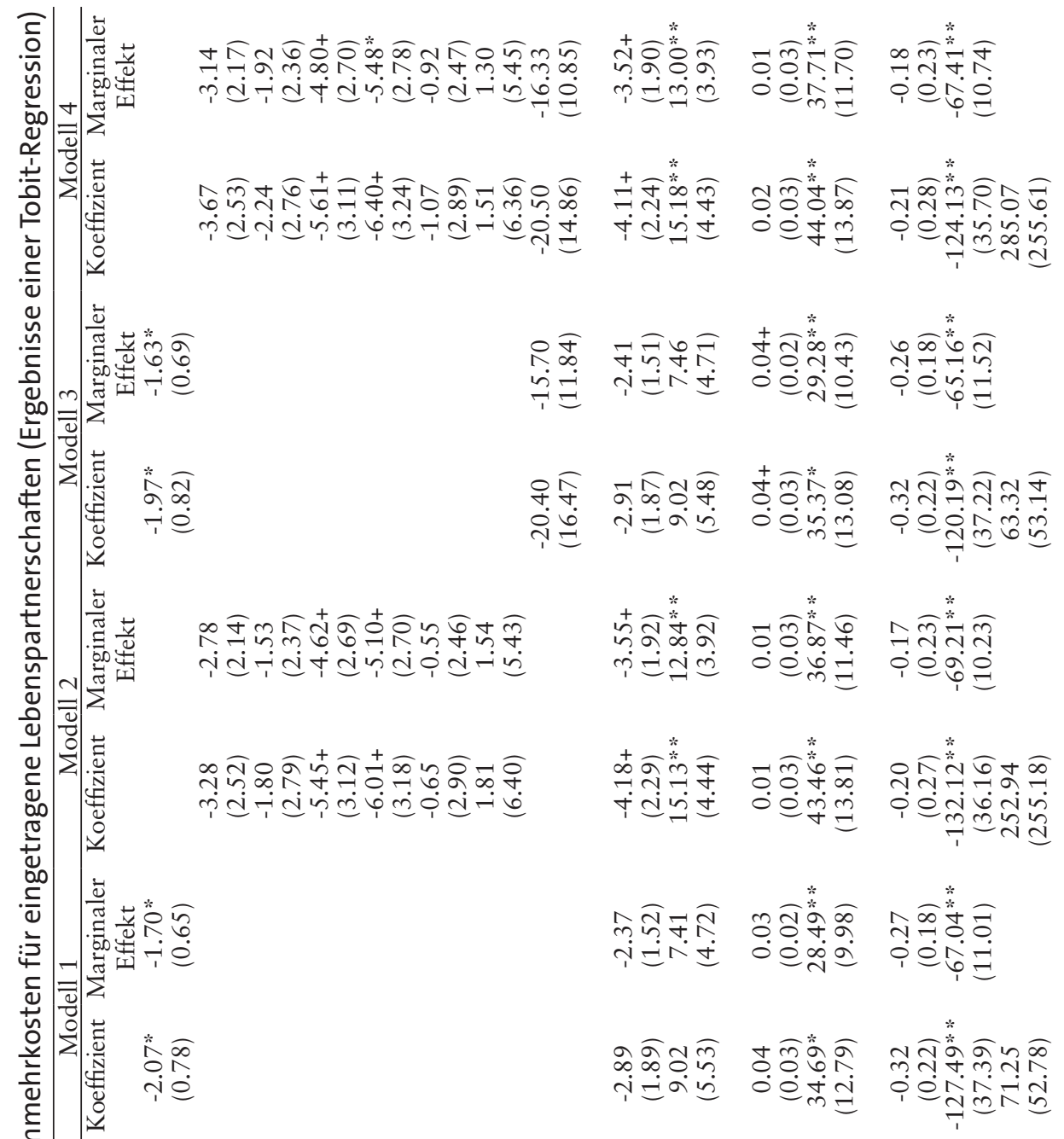




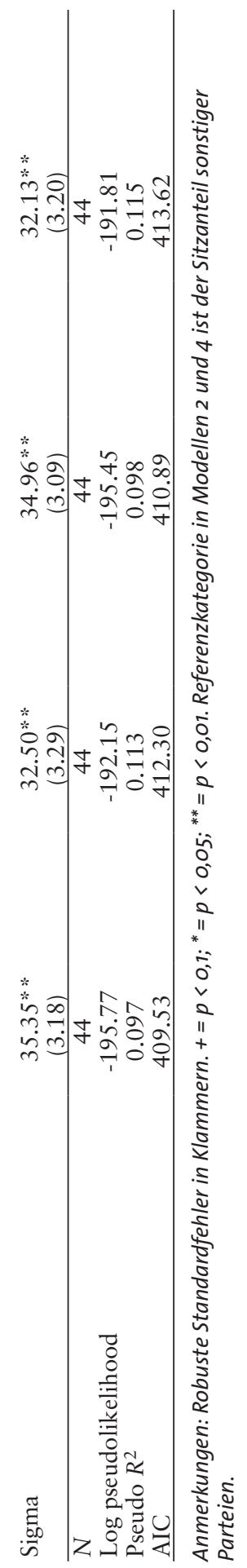




\section{Schlussbetrachtung}

In dieser Abhandlung haben wir uns mit der Varianz in den Gebühren für die Eintragung als Lebenspartnerschaft in Baden-Württemberg beschäftigt. Als mittlerweile einziges deutsches Bundesland - vorbehaltlich einer Änderung durch die im Frühjahr 2011 neu ins Amt gewählte grün-rote Landesregierung - werden Lebenspartnerschaften nicht von Standesbeamten auf Gemeindeebene, sondern von Verwaltungsbeamten der Land- und Stadtkreise geschlossen. Unsere empirischen Analysen haben gezeigt, dass die Gebührenhöhe in Baden-Württemberg zwischen Landkreisen und kreisfreien Städten variiert und diese Variation unter anderem von der Stärke der gesellschaftspolitisch progressiven Parteien und hier insbesondere von den Sitzanteilen von FDP und Bündnis 90/Die Grünen abhängt, selbst wenn für andere Faktoren kontrolliert wird. Zudem gehen deutliche Effekte von weiteren Faktoren, die den ökonomischen Problemdruck und die regionale politische Kultur reflektieren, auf die Höhe der Gebühren, die bei der Schließung einer Lebenspartnerschaft in Baden-Württemberg anfallen, in den 44 Land- und Stadtkreisen aus.

Diese Befunde sind dahingehend interessant, als dass sie in einem Spannungsverhältnis zu den öffentlich-rechtlichen Grundsätzen der Äquivalenz und der Kostendeckung stehen. Tatsächlich können wir davon ausgehen, dass die Kosten für einen solchen Verwaltungsakt unabhängig von der betroffenen regionalen Einheit weitgehend gleich und somit nur geringfügig fluktuieren sollten. Stattdessen konnte aber beobachtet werden, dass lokale und regionale Kontextfaktoren sowie die Präferenzen der parteipolitischen Akteure bei der Festsetzung von Gebührensätzen in dieser moralpolitisch umstrittenen Policy eine zentrale Rolle spielen, was den Grundsatz der Kostendeckung verletzt und Bedenken hinsichtlich des Äquivalenzprinzips nährt. Für diese Sichtweise konnten wir im Zuge unserer Datenrecherche weitere Belege finden. So wurde uns vonseiten einiger Landkreise telefonisch explizit mitgeteilt, dass eine interne Anweisung ergangen sei, die Gebührenordnungen derart zu verändern, dass sie dem Kostendeckungsprinzip schlussendlich entsprechen. Konkret ging dies mit einer Senkung der Gebühren für die Eintragung als Lebenspartnerschaft einher.

Wir sind der Ansicht, dass eine mögliche „Politisierung“ von Gebühren im Speziellen als auch die Rolle der Verwaltung bei parteipolitisch oder ideologisch konfliktären Themen allgemein einen wichtigen - gleichwohl bisher kaum beachteten - Aspekt der Analyse des politischen System Deutschlands sowie der Politikgestaltung darstellen und systematischer untersucht werden sollten. Hieran knüpfen sich Überlegungen hinsichtlich der politischen Steuerungswirkung von Verwaltungsgebühren an, die ebenfalls bislang nur wenig Beachtung gefunden haben. So variiert beispielsweise die Handhabung des Kirchenaustritts zwischen den Bundesländern: Während in Berlin und Brandenburg der Austritt aus einer Kirche gebührenfrei vor dem Amtsgericht erklärt werden kann, erhebt das Land Hessen für die Bearbeitung beim Amtsgericht eine Gebühr von $25 €$. In Schleswig-Holstein hingegen ist - wie auch in Baden-Württemberg oder Bayern - das Standesamt zuständig. Während die Gebühr im nördlichsten deutschen Bundesland $10 €$ und in Bayern $31 €$ beträgt, variiert sie im Südwesten der Republik zwischen 10 
und $60 €$, da in Baden-Württemberg die Gemeinden und nicht das Land die Gebührenhöhe bestimmen.

Ein Anreiz für die weitere Forschung könnte sein, die Gründe für diese von Bundesland zu Bundesland unterschiedlichen Regelungen und Gebührenhöhen herauszuarbeiten. Hierbei können - wie wir im vorliegenden Beitrag zu zeigen versucht haben - theoretische Ansätze aus der Policy-Analyse und der politischen Ökonomie, die auch die Rolle der Verwaltung im politischen System hervorheben, hilfreich sein. Ebenfalls können Ansätze der politischen Kulturforschung, die die symbolische Wirkung politischen Handelns und Entscheidens thematisieren (vgl. etwa Sarcinelli 1989; Edelman 2005; Göhler et al. 2010), fruchtbare Ansätze zum Erklären solcher überraschender Politikergebnisse bieten. Wir hoffen, dass wir mit der vorliegenden Abhandlung die Relevanz dieses speziellen Forschungsdesiderats verdeutlichen und auf diese Weise einen Anstoß für eine umfassendere wissenschaftliche Auseinandersetzung mit der möglichen Politisierung von Verwaltungsvorgängen und der ideologisch determinierten Komponente von Gebühren geben konnten.

\section{Literatur}

Ainsworth, Scott H., und Thad E. Hall. 2010. Abortion Politics in Congress: Strategic Incrementalism and Policy Change. Cambridge: Cambridge University Press.

Bandelow, Nils C. 2003. Lerntheoretische Ansätze in der Policy-Forschung. In Politik als Lernprozess?, Hrsg. Matthias L. Maier, Frank Nullmeier und Tanja Pritzlaff, 98-121. Opladen: Leske + Budrich.

Bennett, Colin J. 1991. What is Policy Convergence and What Causes It? British Journal of Political Science 21:215-233.

Biegelbauer, Peter. 2007. Ein neuer Blick auf politisches Handeln: Politiklernansätze im Vergleich. Österreichische Zeitschrift für Politikwissenschaft 3:231-247.

Boele-Woelki, Katharina. 2008. Legal Recognition of Same-Sex Relationships Within the European Union. Tulane Law Review 82:1949-1981.

Bogumil, Jörg, und Werner Jann. 2009. Verwaltung und Verwaltungswissenschaft in Deutschland - Einführung in die Verwaltungswissenschaft. Wiesbaden: VS Verlag für Sozialwissenschaften.

Bräuninger, Thomas. 2005. A Partisan Model of Government Expenditure. Public Choice 125:409-429.

Bräuninger, Thomas, und Marc Debus. 2008. Der Einfluss von Koalitionsaussagen, programmatischen Standpunkten und der Bundespolitik auf die Regierungsbildung in den deutschen Ländern. Politische Vierteljahresschrift 49:309-338.

Bräuninger, Thomas, und Marc Debus. 2012. Parteienwettbewerb in den deutschen Bundesländern. Wiesbaden: VS Verlag für Sozialwissenschaften.

Bruns, Manfred, und Rainer Kemper. 2005. Lebenspartnerschaftsrecht: Handkommentar. Baden-Baden: Nomos.

Bündnis 90/Die Grünen, und SPD Baden-Württemberg. 2011. Der Wechsel beginnt. Koalitionsvertrag zwischen Bündnis 90/Die Grünen, und SPD Baden-Württemberg. http:// www.gruene-bw.de/fileadmin/gruenebw/dateien/Koalitionsvertrag-web.pdf; zugegriffen 2.8.2011. 
Burgi, Martin. 2010. Verwaltungsorganisationsrecht. In Allgemeines Verwaltungsrecht, Hrsg. Hans-Uwe Erichsen und Dirk Ehlers, 254-327. Berlin: De Gruyter Lehrbuch.

Christlich Demokratische Union Thüringen und Sozialdemokratische Partei Deutschlands, Landesverband Thüringen. 2009. Starkes Thüringen - innovativ, nachhaltig, sozial und weltoffen. Erfurt.

Christlich-Soziale Union und Freie Demokratische Partei Bayern. 2008. Koalitionsvereinbarung 2008 bis 2013 zwischen CSU und FDP für die 16. Wahlperiode des Bayerischen Landtags. München.

Cohen, Jessica, und Denise Langenhan. 2009. Steuerung durch Symbole. In Weiche Steuerung. Studien zur Steuerung durch discursive Praktiken, Argumente und Symbole, Hrsg. Gerhard Göhler, Ulrike Höppner und Sybille De La Rosa, 138-188. BadenBaden: Nomos.

Cusack, Thomas, und Pablo Beramendi. 2006. Taxing Work. European Journal of Political Research 45:43-73.

Die Tageszeitung. 2010. Homo-Ehe in Baden-Württemberg: Trauung im Hinterzimmer. 29.07.2010: http://www.taz.de/1/leben/alltag/artikel/1/trauung-im-hinterzimmer/.

Downs, Anthony. 1957. An Economic Theory of Democracy. New York: Harper.

Edelman, Murray. 2005. Politik als Ritual: Die symbolische Funktion staatlicher Institutionen und politischen Handelns. Frankfurt a. M.: Campus Verlag.

Fink, Simon. 2007. Ein deutscher Sonderweg? Die deutsche Embryonenforschungspolitik im Licht international vergleichender Daten. Leviathan 1:107-128.

Fink, Simon. 2008. Forschungspolitik zwischen Innovation und Lebensschutz. Die Determinanten von Embryonenforschungspolitiken im internationalen Vergleich. BadenBaden: Nomos.

Gammerl, Benno. 2010. Eine Regenbogengeschichte. Aus Politik und Zeitgeschichte 1516:7-13.

Göhler, Gerhard. 2005. Symbolische Politik, symbolische Praxis. Zum Symbolverständnis in der deutschen Politikwissenschaft. In Was heißt Kulturgeschichte des Politischen? Hrsg. Barbara Stollberg-Rillinger, 57-69. Berlin: Duncker und Humblot.

Göhler, Gerhard, Ulrike Höppner, Sybille De La Rosa und Stefan Skupien. 2010. Steuerung jenseits von Hierarchie: Wie diskursive Praktiken, Argumente und Symbole steuern können. Politische Vierteljahresschrift 51:691-720.

Haider-Markel, Donald P. 1999. Morality Policy and Individual-Level Political Behavior. The Case of Legislative Voting on Lesbian and Gay Issues. Policy Studies Journal 27:735-749.

Haider-Markel, Donald P., und Kenneth J. Meier. 1996. The Politics of Gay and Lesbian Rights: Expanding the Scope of Conflict. Journal of Politics 58:332-349.

Haunss, Sebastian. 2004. Identität in Bewegung: Prozesse kollektiver Identität bei den Autonomen und in der Schwulenbewegung. Wiesbaden: VS Verlag für Sozialwissenschaften.

Hall, Peter A. 1993. Policy Paradigms, Social Learning, and the State. Comparative Politics 25:275-296.

Hibbs, Douglas. 1977. Political Parties and Macroeconomic Policy. American Political Science Review 71:1467-1487. 
Holtmann, Everhard. 2002. Parteien und Wählergruppen in der Kommunalpolitik. In Parteiendemokratie in Deutschland, Hrsg. Oscar W. Gabriel und Oskar Niedermayer, 408427. Wiesbaden: VS Verlag für Sozialwissenschaften.

Holtmann, Everhard. 2008. „Parteifrei“ aus Überzeugung und aus Verlegenheit - Einleitende Bemerkungen zu Präsenz und Profil kommunaler Wählergemeinschaften in Deutschland. In Parteifrei im Parteienstaat - Kommunale Wählergemeinschaften: Elektorale Verankerung und soziales Profil im Ost-West-Vergleich, Mitteilungen des SFB 580 Nr. 25, Hrsg. Everhard Holtmann, 6-9. Halle: SFB 580.

Holzinger, Katharina. 2009. Vom ungeliebten Störenfried zum akzeptierten Paradigma? Zum Stand der (neuen) politischen Ökonomie in Deutschland. Politische Vierteljahresschrift 50:539-576.

Knill, Christoph. 2006. Implementation. In European Union. Power and Policy-Making, Hrsg. Jeremy Richardson, 351-375. London: Routledge.

Knill, Christoph, und Jale Tosun. 2010. Politikgestaltung in der Europäischen Union. Die Entwicklung und Umsetzung der Dienstleistungsrichtlinie. Baden-Baden: Nomos.

Knill, Christoph, Katharina Holzinger und Helge Jörgens. 2007. Transfer, Diffusion und Konvergenz: Konzepte und Kausalmechanismen. In Transfer, Diffusion und Konvergenz von Politiken, PVS Sonderheft 38, Hrsg. dies., 11-38. Wiesbaden: VS Verlag für Sozialwissenschaften.

Landtag von Baden-Württemberg. 2010. Zweite Beratung des Gesetzentwurfs der Landesregierung - Gesetz zur Reform des öffentlichen Dienstrechts (Dienstrechtsreformgesetz - DRG) - Drucksache 14/6694. In Plenarprotokoll 14 / 102 vom 27. 10. 2010, 72517271. Stuttgart: Landtag von Baden-Württemberg.

Lipset, Seymour M., und Stein Rokkan. 1967. Cleavage Structures, Party Systems and Voter Alignments. An Introduction. In Party Systems and Voter Alignments. Cross-National Perspectives, Hrsg. Seymour M. Lipset und Stein Rokkan, 1-64. New York/London: Free Press.

Lütz, Susanne. 2002. Der Staat und die Globalisierung von Finanzmärkten. Regulative Politik in Deutschland, Großbritannien und den USA. Frankfurt a. M.: Campus Verlag.

Magin, Raphael, Markus Freitag und Adrian Vatter. 2009. Cleavage Structures and Voter Alignments Within Nations: Explaining Electoral Outcome in Germany's Counties, 1998 to 2005. Zeitschrift für Vergleichende Politikwissenschaft 3:231-256.

May, Peter J. 2003. Policy Design and Implementation. In Handbook of Public Administration, Hrsg. Guy B. Peters und Jon Pierre, 223-233. Thousand Oaks: Sage.

Mayntz, Renate. 1983. Implementation politischer Programme II - Ansätze zur Theoriebildung. Opladen: Westdeutscher Verlag.

Mayntz, Renate. 1990. Politische Steuerbarkeit und Reformblockaden: Überlegungen am Beispiel des Gesundheitswesens. Staatswissenschaft und Staatspraxis 1:283-307.

Mayntz, Renate, und Fritz W. Scharpf. 2005. Politische Steuerung - Heute? Zeitschrift für Soziologie 34:236-243.

Meier, Kenneth J. 1994. The Politics of Sin. Drugs, Alcohol and Public Policy. Armonk: Sharpe.

Moeller, Robert G. 1994. The Homosexual Man is a 'Man', the Homosexual Woman is a Woman: Sex, Society, and the Law in Postwar West Germany. Journal of the History of Sexuality 4:395-429. 
Mooney, Christopher Z. 2001. The Public Clash of Private Values: the Politics of Morality Policy. New York: Chatham House.

Mooney, Christopher Z., und Mei-Hsien Lee. 2000. The Influence of Values on Consensus and Contentious Morality Policy: U.S. Death Penalty Reform, 1956-82. The Journal of Politics 62:223-239.

Mooney, Christopher Z., und Richard G. Schuldt. 2008. Does Morality Policy Exist? Testing a Basic Assumption. The Policy Studies Journal 36:199-218.

Mueller, Dennis. 2003. Public Choice III. Cambridge: Cambridge University Press.

Müller, Jochen. 2009. The Impact of the Socio-Economic Context on the Länder Parties' Policy Positions. German Politics 18:365-384.

Niskanen, William A. 1971. Bureaucracy and Representative Government. Chicago: Aldine-Atherton.

Oberndörfer, Dieter, und Karl Schmitt. 1991. Parteien und regionale politische Traditionen in Deutschland. Berlin: Duncker \& Humblot.

Pappi, Franz U., und Susumu Shikano. 2002. Die politisierte Sozialstruktur als mittelfristig stabile Basis einer deutschen Normalwahl. Kölner Zeitschrift für Soziologie und Sozialpsychologie 54:444-475.

Pappi, Franz U., und Susumu Shikano. 2004. Ideologische Signale in den Wahlprogrammen der deutschen Bundestagsparteien 1980 bis 2002. MZES Working Papers Nr. 76. Mannheim: Mannheimer Zentrum für Europäische Sozialforschung.

Patton, Dana. 2007. The Supreme Court and Morality Policy Adoption in the American States. Political Research Quarterly 60:468-488.

Pickel, Susanne und Gert Pickel. 2006. Politische Kultur- und Demokratieforschung. Grundbegriffe, Theorien, Methoden. Eine Einfübrung. Wiesbaden: VS Verlag für Sozialwissenschaften.

Powell, G. Bingham. 2000. Elections as Instruments of Democracy: Majoritarian and Proportional Visions. London: New Haven.

Powell, G. Bingham. 2004. The Chain of Responsiveness. Journal of Democracy 15:91105.

Pressman, Jeffrey, und Aaron Wildavsky. 1973. Implementation. Berkeley: University of California Press.

Reiser, Marion, und Adrienne Krappidel. 2008. Parteien ohne Parteilichkeit? Analyse zum Profil parteifreier Gruppierungen. In Parteifrei im Parteienstaat - Kommunale Wählergemeinschaften: Elektorale Verankerung und soziales Profil im Ost-West-Vergleich, Mitteilungen des SFB 580 Nr. 25, Hrsg. Everhard Holtmann, 74-102. Halle: SFB 580.

Rohe, Karl. 1992. Wablen und Wählertraditionen. Kulturelle Grundlagen deutscher Parteien und Parteiensysteme im 19. und 20. Jahrhundert. Frankfurt a. M.: Suhrkamp.

Rohe, Karl. 1996. Politische Kultur: Zum Verständnis eines theoretischen Konzepts. In Politische Kultur in Ost- und Westdeutschland, Hrsg. Oskar Niedermayer und Klaus von Beyme, 1-21. Opladen: Leske + Budrich.

Sabatier, Paul A. 1993. Advocacy-Koalitionen, Policy-Wandel und Policy-Lernen: Eine Alternative zur Phasenheuristik. In Policy-Analyse. Kritik und Neuorientierung, Hrsg. Adrienne Héritier, 116-148. Opladen: Westdeutscher Verlag.

Sarcinelli, Ulrich. 1989. Symbolische Politik und politische Kultur. Das Kommunikationsritual als politische Wirklichkeit. Politische Vierteljahresschrift 30: 292-309. 
Scharpf, Fritz W. 1989. Politische Steuerung und politische Institutionen. Politische Vierteljahresschrift 30:10-21.

Schmidt, Manfred. 1996. When Parties Matter: A Review of the Possibilities and Limits of Partisan Influence on Public Policy. European Journal of Political Research 30:155183.

Schmitt-Beck, Rüdiger. 2000. Die hessische Landtagswahl vom 7. Februar 1999: Der Wechsel nach dem Wechsel. Zeitschrift für Parlamentsfragen 31:3-17.

Schmitt-Beck, Rüdiger, und Thorsten Faas. 2009. Die hessische Landtagswahl vom 27. Januar 2008: Wiederkehr der „hessischen Verhältnisse“. Zeitschrift für Parlamentsfragen 40:16-34.

Schnapp, Kai-Uwe. 2004. Ministerialbürokratien in westlichen Demokratien. Opladen: Leske + Budrich.

Shepsle, Kenneth A., und Mark S. Bonchek. 1997. Analyzing Politics: Rationality, Behavior and Institutions. New York/London: Norton + Company.

Sommerer, Thomas. 2010. Können Staaten voneinander lernen? Eine vergleichende Analyse der Umweltpolitik in 24 Ländern. Wiesbaden: VS Verlag für Sozialwissenschaften. Stadtnachrichten Rottweil. 2010. Stadt passt Steuern und Gebühren an. Räte beschließen Maßnahmen zur Haushaltskonsolidierung. 25.11.2010: http://www.rottweil.de/ceasy/ modules/cms/main.php5?cPageId=1983\&view=publish\&item=article\&id=1012.

Statistisches Landesamt Baden-Württemberg (Hrsg.). 1997. Statistik von Baden-Württemberg, Band 518, Die Bevölkerung 1996. Stuttgart: Statistisches Landesamt BadenWürttemberg.

Stecker, Christian. 2011. Bedingungsfaktoren der Fraktionsgeschlossenheit. Eine vergleichende Analyse der deutschen Länderparlamente. Politische Vierteljahresschrift 52:424-446.

Steinke, Ron. 2005. Ein Mann, der mit einem anderen Mann... - Eine kurze Geschichte des $\mathbb{1 7 5}$ in der BRD. Forum Recht 2:60-63.

Strøm, Kaare, und Wolfgang C. Müller. 1999. Political Parties and Hard Choices. In Policy, Office, or Votes? How Political Parties in Western Europe Make Hard Decisions, Hrsg. Wolfgang C. Müller und Kaare Strøm, 1-35. Cambridge: Cambridge University Press.

Stümke, Hans-Georg. 1989. Homosexuelle in Deutschland: Eine politische Geschichte. München: Beck.

Stümke, Hans-Georg, und Rudi Finkler. 1981. Rosa Winkel, rosa Listen: Homosexuelle und Gesundes Volksempfinden. Von Auschwitz bis heute. Reinbek: Rowohlt.

Süddeutsche Zeitung. 2010. Gebühren: Schwule berappen mehr. 10.08.2010: http://www. sueddeutsche.de/geld/gebuehren-schwule-berappen-mehr-1.986481.

Tatalovich, Raymond, und Alexander T. Smith. 2001. Status Claims and Cultural Conflicts: The Genesis of Morality Policy. Policy Currents 10:2-8.

Völkl, Kerstin, Kai-Uwe Schnapp, Everhard Holtmann und Oscar W. Gabriel. 2008. Wähler und Landtagswablen in der Bundesrepublik Deutschland. Baden-Baden: Nomos.

Waaldijk, Kees. 2004. Others May Follow: the Introduction of Marriage, Quasi-Marriage, and Semi-Marriage for Same-Sex Couples in European Countries. New England Law Review 38:569-589. 
Wagschal, Uwe, und Georg Wenzelburger. 2009. Determinanten der Haushaltskonsolidierung der Bundesländer (1992-2006). Zeitschrift für Vergleichende Politikwissenschaft 3:33-58.

Wald, Kenneth D., James W. Button und Barbara A. Rienzo. 1996. The Politics of Gay Rights in American Communities: Explaining Antidiscrimination Ordinances and Policies. American Journal of Political Science 40:1152-1178.

Wegrich, Kai. 2006. Steuerung im Mehrebenensystem der Länder: Governance-Formen zwischen Kooperation, Hierarchie und Management. Wiesbaden: VS Verlag für Sozialwissenschaften.

Wehling, Hans-Georg. 2006. Föderalismus und politische Kultur in der Bundesrepublik Deutschland. In Landespolitik in Deutschland: Grundlagen - Strukturen - Arbeitsfelder, Hrsg. Herbert Schneider und Hans-Georg Wehling, 87-107. Wiesbaden: VS Verlag für Sozialwissenschaften.

Winter, Søren C. 2003. Implementation Perspectives: Status and Reconsideration. In Handbook of Public Administration, Hrsg. Guy B. Peters und Jon Pierre, 212-222. Thousand Oaks, London: Sage.

\section{Autorenangaben:}

Prof. Dr. Marc Debus,

Carl von Ossietzky Universität Oldenburg, Institut für Sozialwissenschaften,

26129 Oldenburg, marc.debus@uni-oldenburg.de

[Korrespondenzautor]

Prof. Dr. Christoph Knill, Universität Konstanz, Fachbereich Politik- und Verwaltungswissenschaft, Lehrstuhl für Vergleichende Policy-Forschung und Verwaltungswissenschaft, Universitätsstraße 10, Fach D 91, 78457 Konstanz, christoph.knill@uni-konstanz.de

Dr. Jale Tosun, Universität Konstanz, Fachbereich Politik- und Verwaltungswissenschaft, Lehrstuhl für Vergleichende Policy-Forschung und Verwaltungswissenschaft, Universitätsstraße 10, Fach D 91, 78457 Konstanz, jale.tosun@uni-konstanz.de 